\title{
8 \\ THE DEVELOPMENT OF A KNOWLEDGE FRAMEWORK THROUGH INNOVATION BETWEEN AN SME AND A MULTINATIONAL CORPORATION
}

\author{
Frank Murray \\ Piercom Limited \\ Shannon, Ireland
}

\begin{abstract} be summarized as follows:

- Be customer centric

- Be agile

- Be knowledge intensive

- Be responsible to worker needs

- Be networked

- Be productive

- Be involved

- Be continually learning

- Be proactively diverse
\end{abstract}

Companies must continue to innovate if they are to remain resilient to changing markets and customer conditions. This is more prescriptive for mature companies where products and innovations may have peaked. Worldclass companies are constantly seeking new ways to innovate and change to remain relevant and competitive. This case study looks at two technology companies, a multinational and an SME, coming from different technology poles, one hardware micro-technology and one software process technology, sharing skillsand knowledge to remain resilient to ever-changing challenges.

The key attributes demonstrated by the companies during this project could

Readers of this paper may identify these attributes as building blocks for resilience as they may provide insights into what a knowledge enterprise should value to remain resilient and resourceful.

Keywords Knowledge framework development, software process improvement, zero defects, measurement

Please use the following format when citing this chapter:

Murray, Frank, 2006, in International Federation for Information Processing (IFIP), Volume 206, The Transfer and Diffusion of Information Technology for Organizational Resilience, eds. B. Donnellan, Larsen T., Levine L., DeGross J. (Boston: Springer), pp. 109-115. 


\section{INTRODUCTION}

Automotive, biomedical, and other vertical markets are insisting on zero defects from their suppliers. Product recalls have serious effects on cost, brand, and customer satisfaction. A standard of less than 10 parts per million failure was acceptable in the past with error responsiveness measured over months and weeks as the norm. In today's market, if errors do occur, then traceability is expected from suppliers within hours of occurrence and close loop remediation cycles within 24 to 48 hours.

Analog Devices Incorporated (ADI), a mixed signal design-to-manufacturer integrated circuit provider, identified the need to extend it's chip technology test coverage compliance to include the software test programs used to test hardware parts, thereby extending their supply-chain interrogation to achieve a holistic response to customer errors. It engaged Piercom, an Irish-based software engineering SME, to apply software process improvement techniques for key performance areas (KPAs) and set up the key performance indicators (KPIs) to trace software test errors. Piercom applied the capability maturity model (1)(CMM) as a process methodology which provides measures in software best practice across people, process, and technology paradigms. The objective of this exercise was to benchmark ADI against an established software process best-practice approach model and evaluate if any gaps in software process contributed to increased errors. The project was initiated as a result of a software test coverage issue reported by an ADI customer. Using CMM, Piercom Limited performed a traceability audit to measure errors. A joint team consisting of ADI and Piercom engineers was set up to capture the information around the software test domain. This team approach resulted in the establishment of a joint innovation, the knowledge framework, that combines processes and information around the software test domain.

\section{THE RESEARCH APPROACH}

The knowledge framework consists of an information repository that warehouses data, information and knowledge and acts as a portal comprising of tools, techniques, procedures, and embedded learning process guidelines in software best practice.

ADI formed a collaborative research project with Piercom seeking to provide knowledge transfer of skills, competence, and software process methods from Piercom to ADI. Piercom were tasked to deliver the knowledge framework infrastructure for combining software processes for the test engineering group at ADI.

The goals for the project were identified as

1. Reduce customer errors

2. Provide a real-time process to identify and remediate problems within $n$ days

3. Establish a framework and best practices to proactively deal with potential errors

4. Build on ADI's investment in skill and knowledge, improving company competitiveness and resilience

An example demonstrates the need for this innovation: 
The automotive industry has set zero defects as a goal for parts shipped by suppliers. An automobile manufacturer has to recall a model due to an error showing in the digital dashboard of a car. The integrated circuit (IC) in the steering harness is suspected of causing the error and the hardware and software solutions are examined. It is demonstrated that the IC failed its test coverage tolerances and for the chip supplied there must be 100 percent trace ability to capture the error in 24 hours. This supply-chain reversal search must identify all tests performed on the IC, all technology tools used, the personnel (trained or otherwise used and certification) to test the devices.

In mixed engineering environments, where hardware and software overlap, audit trails through people skills, training, mentorship, technology, processes, and procedures are a critical part of remediation.

This program seeks to create the measurement dashboard and intellectual property framework that harnesses this knowledge by development of a knowledge framework best practice.

\section{THE KNOWLEDGE FRAMEWORK PROJECT}

Customer error metrics were gathered. These were traced through development of people, process, and technology measures or KPIs. Reviews were conducted with a cross section of engineers to ascertain the skill, support, and certification for the testengineering faculty in the exercise of their roles. Code previews and reviews were established. The development of coding practices, code reuse methodologies, and requirement to retain information pointed to a need for an open portal or IT infrastructure to warehouse and manage the different code components, third party tools, research, and best practice process reviews.

Project Phase 1 (120 Days): Define and implement the CMM process methodologies for a group of product lines measuring customer errors. Scope the needs for the high level architecture of the process workflows across the product test lifecycle.

Project Phase 2 (120 Days): Implement across a wider range of product lines and develop the knowledge framework portal infrastructure to manage the shared services knowledge pool.

\section{THE SOLUTION}

Piercom Limited selected CMM as a benchmark process to evaluate Analog Devices' level of compliance to the standards set out under CMM's levels 1 through 5 .

Metrics were gathered to evaluate customer errors, time to market, and new product introduction process steps. This forms the "management dashboard" to provide control and measurement to executive management. Reviews were conducted with a cross section of engineers to ascertain the skills and support structures available to the test engineering faculty in the exercise of their roles. A central team was selected from both companies to ensure that ownership and structure were established across process, skills, 
technology tools, and IT infrastructure. Piercom Limited provided parallel resources to coach and guide such areas as code previews and reviews, development of coding practices, code reuse opportunity, and IT infrastructure in database design, third party tools research, and best practice process reviews. A complete rewrite of the overall testing (hardware and software) roadmap incorporating software linked steps was undertaken.

\section{THE RESULTS}

A business model and architecture called the knowledge framework for testing practices emerged. Customer communication became more proactive as a result of data combined in the knowledge framework infrastructure and measurement around error tracking improved. There was increased emphasis on interlinked IT networks and databases. A more concentrated effort to profile skills and training needs was a significant by-product of the project. A skills matrix for future learning was developed. Different agile coding practices and formal methods were examined and a "cookbook" developed to implement consistent coding convention. KPIs and KPAs were clearly identified and measured. There was a marked improvement in process and procedural steps in software testing.

\section{KEY RECOMMENDATIONS FROM THE PROJECT}

It was recommended that $\mathrm{CMM}$ be adopted as the benchmark process to maintain the control model for the testing lifecycle as it pertains to software. The implementation of a formal certified training and mentorship program was also identified. Training of all engineers in CMM awareness and the software lifecycle model would be a key valueadded knowledge investment. Establishing a skills matrix as a key component of test project definition was also identified and recommended It was felt that there was a reduction in new product slippage due to software process improvement and it was recommended that the program be extended to 15 product lines. The development of a reusable-software database was seen as key to continuous improvement. The institution of consistent project management procedures, coding methodologies, and naming conventions was seen as a sensible approach to reducing repetition and inefficiency. The building of a consistent knowledge infrastructure to support a software reuse methodology and optimize the use of tools and software components was a key recommendation. The project gave ADI and Piercom the ability to track the percentage of code reuse per derivative, platform, and breakthrough new product release and was accepted by all as key to successful management of test error remediation.

Finally, the development of external linkages with other companies and academic institutions for shared learning was seen as important for insights into process innovation and learning. This had been a blind spot in benchmarking best practice. Utilizing an SME as a catalyst to focus effort in a specific knowledge domain was seen as a good first step in innovative resilience. 


\section{FUTURE COLLABORATION AND EXPANSION OF KNOWLEDGE FRAMEWORK}

Piercom and ADI have identified the need for such a knowledge framework infrastructure and have proceeded commercially to develop this project. The opportunity to map the needs of other multinationals into such a program seems logical to benefit a wider audience. If the needs analysis and mapping was completed across a group of multinationals, then the opportunity to provide a generic knowledge framework could serve this domain across industry.

ADI and Piercom are keen to develop this initiative with partners providing the knowledge framework approach for advanced learning and skills development. The joint project team have visited other multinationals across different vertical markets to evaluate if the needs at ADI are mirrored for these other multinationals in the test practices domain. The findings are that there is similarity and opportunity for best practices through process innovation concepts to scale across vertical markets (automotive, semiconductor, and bio-medical). Indeed the development of such a best practice competency could leverage competition for all multinationals. Harnessing existing competency and building on existing platforms of knowledge can prove a decisive competitive tool in expanding existing mandates, which could be argued is a driver of resilience.

The testing arena could become a key differentiator for ADI and the raw material (resource) is in place through the test engineering resources group, whose skill and knowledge can be harnessed.

ADI and Piercom have invested significant hours in meetings and discussions with seven multinationals (IBM, Avocent, Lucent, Kostal, Boston Scientific, Nortel, and Analog Devices), presenting a proof of concept model and receiving formal support from four multinationals to progress to a cluster in this area. The seven multinationals have indicated their interest in progressing further in 2006 . They believe that they could champion the case within their corporations for collaboration and shared-learning in this area.

ADI, the Atlantic Technology Corridor (an Irish association promoting the development of industry and technology along the Atlantic coast) support the cluster. All of the companies listed above have agreed to lend their names to this initiative, as have academic and government agencies.

\section{APPROACH USED IN FUTURE COLLABORATION}

To develop a cluster in this area, the aproach used will be similar to that outlined in ECOLEAD, a European Consortia using virtual organization partnerships to develop linkages for shared learning between companies and academia.

The associated collaborative cluster consists of multinationals, small to medium enterprises, and research groups. Value-added is through a shared knowledge model. The work could lead to the development of an international competency cluster in test best practices and leverage other research and IP creation. 


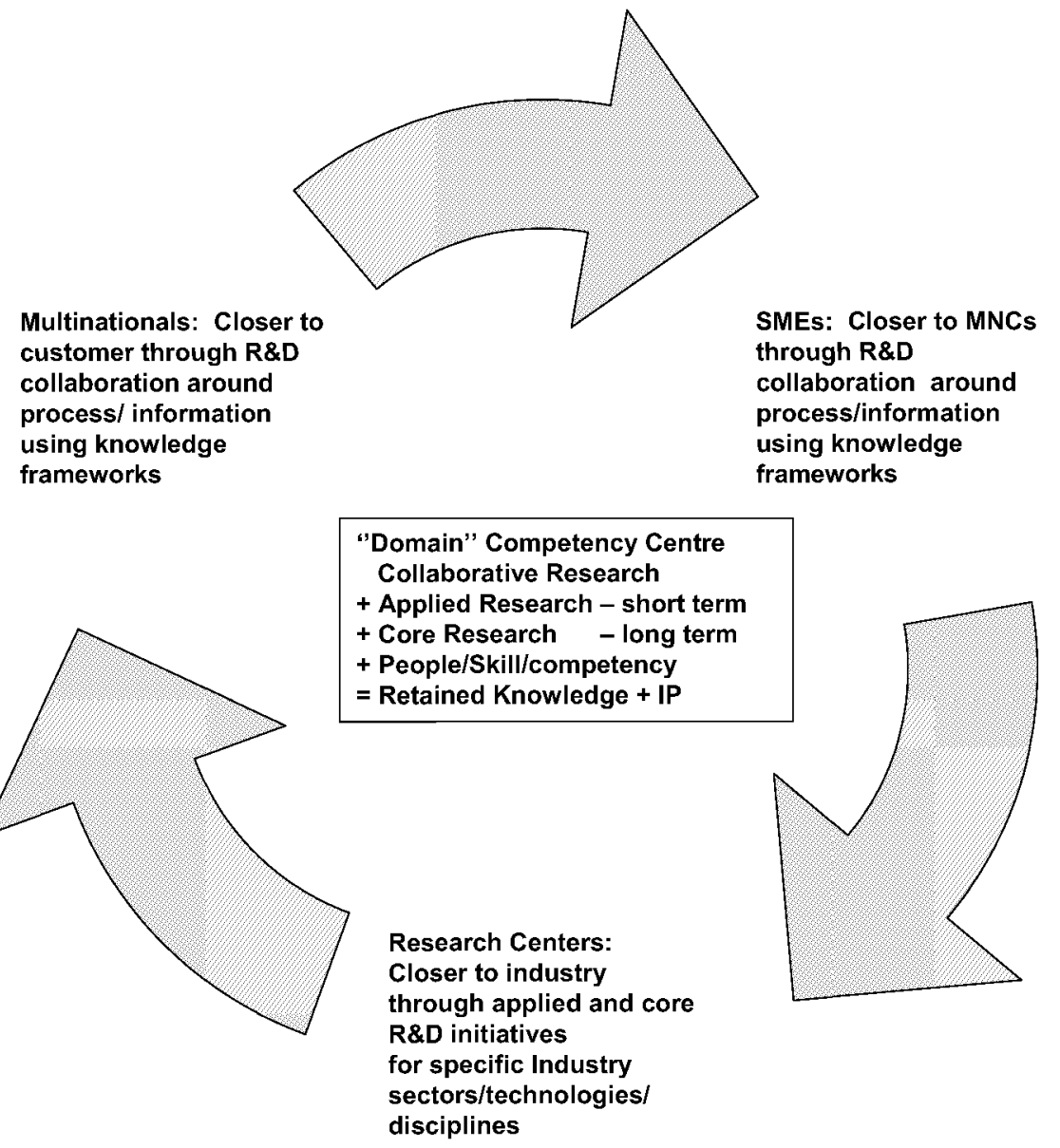

Figure 1. Collaborative Groups in Proposed Competency Cluster Supported by a Knowledge Framework. (Source: Piercom Limited \& Analog Devices Corporation Project Team)

\section{Acknowledgments}

The author wishes to thank Dr. Brian Donnellan, NUIG Galway for inviting Piercom and Analog Devices to share their experiences. The author would also like to acknowledge the support of Dick Meaney, Vice President, ADI, J. J. O'Riordan, Head of Test \& Measurement, ADI, Gordon Thomson, Head of Quality Test Practices, ADI, Paul Newton, Software Process Director, Piercom Limited, and Paul Ryan, Head of Process Consulting, Piercom Limited, for their significant participation in this program. The support of IDA, Ireland, Enterprise Ireland, Norte1, Boston Scientific, and Kostal GMBH is also acknowledged. 


\section{About the Author}

Frank Murray has over 25 years of international software business management experience working for the multinational and SME sectors in the United States and Europe. Frank has been Managing Director of Piercom Limited from 1998 and is the majority owner. Frank held senior executive roles as European Managing Director for the Briggs \& Stratton software division and as principal at DFS Associates, the Swiss-based consultancy specializing in software innovation. As European Software Business Practices Manager for Digital Equipment Corporation (later CompaQ and HP), Frank managed operations for DEC's 1Bn software enterprise. In 2004, Frank was invited by Professor David Parnas to participate on the Advisory Board for the Software Quality Reliability Laboratory at University of Limerick.

Frank was awarded a Bachelor of Science degree in Mathematics, Analytical Techniques and Instrumentation and Chemistry from NUIG in 1979. He attended the International Advanced Management Program at INSEAD, Paris; IMD, Lausanne and IFL in Sweden from 1984-1989. He serves on a number of commercial and voluntary management boards and has delivered a number of keynote addresses to international conferences and to academic institutes. Frank can be reached by e-mail at frank.murray@piercom.ie.

\section{Affiliations: Piercom Limited}

Piercom Limited are a provider of best practice process improvement innovation techniques. The research and development culture at Piercom has led to the creation of a number of innovative solutions to solve large IT integration issues across people, process, and technology. Piercom are developing the capability to deliver the knowledge infrastructure that supports software best practices.

In 2002, Piercom Limited provided the CRM solutions to the Irish government that resulted in Ireland achieving the number 1 position in e-government benchmarking across 18 countries as reported by Cap Gemini. In 1998, Piercom Limited were awarded the prestigious Washingtonbased Smithsonian Medal for Innovation in Technology and business-related services. Piercom's technology is showcased at the institute.

Piercom Limited are the IT partners of the Irish government and list General Electric Aviation, ADI, Dell, Banta, Johnson \& Johnson, Nortel, Boston Scientific, and Kostal GMBH as part of their growing network of clients. Piercom Limited are members of LERO, the Irish Software Engineering Research Centre recently formed through Science Foundation Ireland funding. The company was founded in 1993 as a campus company at the University of Limerick and has evolved from software reengineering through IT integration and into software process.

Analog Devices, Inc. (NYSE: ADI) is a world-leading semiconductor company specializing in high performance analog, mixed-signal, and digital signal processing integrated circuits. Since ADI was founded in 1965, its focus has been to solve the engineering challenges associated with signal processing in electronic equipment. ADI's products play a fundamental role in converting real-world phenomena such as temperature, motion, pressure, light, and sound into electrical signals to be used in a wide array of applications ranging from industrial process control, factory automation, radar systems, and CAT scanners to cellular base stations and telephones, broadband networking, computers, cars, and digital cameras.

ADI currently has a worldwide workforce of approximately 8,900 employees, including over 3,000 engineers. Wafer fabrication facilities for high-performance analog products are located in Massachusetts, California, and Ireland. Product testing is conducted at facilities in the Philippines. Corporate headquarters are located in Norwood, Massachusetts. 